\title{
Does $\Lambda$ CDM really be in tension with the Hubble diagram data?
}

\author{
Ahmad Mehrabi $^{1, \mathrm{a}} \mathbb{1}$, , Spyros Basilakos ${ }^{2, \mathrm{~b}}$ \\ ${ }^{1}$ Department of Physics, Bu-Ali Sina University, Hamedan 65178 016016, Iran \\ 2 Academy of Athens, Research Center for Astronomy and Applied Mathematics, Soranou Efessiou 4, 11-527 Athens, Greece
}

Received: 22 February 2020 / Accepted: 9 July 2020 / Published online: 16 July 2020

(C) The Author(s) 2020

\begin{abstract}
In this article, we elaborate further on the $\Lambda \mathrm{CDM}$ "tension", suggested recently by the authors Lusso et al. (Astron Astrophys 628:L4, 2019) and Risaliti and Lusso (Nat Astron 3(3):272, 2019). We combine Supernovae type Ia (SNIa) with quasars (QSO) and Gamma Ray Bursts (GRB) data in order to reconstruct in a model independent way the Hubble relation to as high redshifts as possible. Specifically, in the case of either SNIa or SNIa/QSO data we find that the current values of the cosmokinetic parameters extracted from the Gaussian process are consistent with those of $\Lambda \mathrm{CDM}$. Including GRBs in the analysis we find a tension, which lies between $2 \sigma$ and $3 \sigma$ levels respectively. Finally, we find that at high redshifts $(z>1)$ the corresponding cosmokinetic parameters significantly deviate from those of $\Lambda \mathrm{CDM}$, hence the possibility of new Physics is not precluded by the present analysis.
\end{abstract}

\section{Introduction}

Since the discovery of the accelerated expansion of the Universe from the Supernovae type Ia (SNIa) data $[3,4]$, the combined analysis of various cosmological probes, including those of Cosmic Microwave Background (CMB) [57], Baryon Acoustic Oscillation (BAO) [8-14] and cosmic chronometers [15] confirms the aforementioned dynamical result, namely that currently the Universe accelerates. However, the physics of cosmic acceleration is still a mystery, hence the aim in these kind of studies is to provide an explanation regarding the underlying mechanism which triggers such a phenomenon.

In the framework of homogeneous and isotropic Universe, the accelerated expansion can be described by considering either an exotic matter with negative pressure [16-22] or a modification of gravity $[f(R)$ theories and the like, 23-

\footnotetext{
a e-mail: Mehrabi@basu.ac.ir (corresponding author)

be-mail: svasil@academyofathens.gr
}

27]. Among the large family of dark energy and modified gravity models, the simplest case is the spatially flat $\Lambda \mathrm{CDM}$ model for which cold dark matter $(\mathrm{CDM})$ and baryonic matter coexist with the cosmological constant. From the theoretical viewpoint, the $\Lambda \mathrm{CDM}$ model suffers from the well known problems, namely the coincidence and the expected value of the vacuum energy density [28-31].

On the other hand, despite the fact that the $\Lambda \mathrm{CDM}$ model is found to be in a very good agreement with the majority of cosmological data [7], nonetheless the model seems to be currently in tension with some recent measurements [32-35], related with the Hubble constant $H_{0}$ and the present value of the mass variance at $8 h^{-1} \mathrm{Mpc}$, namely $\sigma_{8}$. Moreover, Lusso et al. [1] using a combined Hubble diagram of SNIa, Quasars, and gamma-ray bursts (GRBs) found $\mathrm{a} \sim 4 \sigma$ tension between the best fit cosmographic parameters with respect to those of $\Lambda \mathrm{CDM}$ (see also [2,36]). In the light of the latter results, a heated debate is taking place in the literature and the aim of the present article is to contribute to this debate.

Here, we focus on a model-independent parametrization of the Hubble diagram using the Gaussian process, and investigate its performance against the latest Hubble diagram data. Notice that in this case we need to introduce a kernel function with some hyperparameters which can be optimized in order to fit the data. For more details concerning modelindependent methods we refer to [37-40]. The structure of the paper is as follows. In Sect. 2, we introduce the concept of the Gaussian process and we present the corresponding kernel functions that we shall use in the current work. In Sect. 3, we discuss the observational data and the procedure of our analysis, while in Sect. 4 we provide our results. Finally, in 5 , we summarize our results and we draw our conclusions.

\section{Model independent method-Gaussian process}

We consider that the universe is a self-gravitating fluid, endowed with a spatially flat homogeneous and isotropic 
geometry. In this context, there are two main approaches in order to investigate cosmological data e.g. the luminosity distance. In the first case we impose a cosmological model, hence we estimate the form of the luminosity distance. Then we fit the model to data in order to place constraints on the corresponding parameter space. This is a model-depended method is a sense that different models provide different forms of luminosity distance. Another avenue is to utilize a model independent method in reconstructing the Hubble diagram through the observational data [37-40]. In this approach we do not need to know apriori the underlying cosmological model. One of the most popular model independent method is the Gaussian process (GP), hence in the present article we test the performance of GP against the available Hubble diagram data.

Briefly, the main steps of the method are the following. Having a data set $D$

$D=\left\{\left(x_{i}, y_{i}\right) \mid i=1, \ldots, n\right\}$,

our aim is to reconstruct in a model independent way a function $f(x)$ which describes the data. In this case at any point $x$, the value $f(x)$ is a Gaussian random variable with mean $\mu(x)$ and variance $\operatorname{Var}(x)$. Moreover, the function values at any two different points are not independent from each other, hence the covariance function $\operatorname{cov}(f(x), f(\tilde{x}))=k(x, \tilde{x})$ describes the corresponding correlations. Therefore, having an observational data set $\left(x_{i}, y_{i}\right)$ and considering a kernel function $k(x, \tilde{x})$, it is straightforward to compute the value of function and its covariance (for more detail see [41]). Concerning the functional form of the kernel, there is a wide range of possibilities. In the current work we restrict our analysis to the following parametrizations:

$$
\begin{aligned}
k(x, \tilde{x})= & \sigma_{f}^{2} \exp \left(-\frac{(x-\tilde{x})^{2}}{2 l^{2}}\right) \\
k(x, \tilde{x})= & \sigma_{f}^{2} \exp \left(-\sqrt{7} \frac{|x-\tilde{x}|}{l}\right)\left(1+\sqrt{7} \frac{|x-\tilde{x}|}{l}\right. \\
& \left.+14 \frac{(x-\tilde{x})^{2}}{5 l^{2}}+7 \sqrt{7} \frac{|x-\tilde{x}|^{3}}{15 l^{3}}\right),
\end{aligned}
$$

and

$$
\begin{aligned}
k(x, \tilde{x})= & \sigma_{f}^{2} \exp \left(-3 \frac{|x-\tilde{x}|}{l}\right)\left(1+3 \frac{|x-\tilde{x}|}{l}+27 \frac{(x-\tilde{x})^{2}}{7 l^{2}}\right. \\
& \left.+18 \frac{|x-\tilde{x}|^{3}}{7 l^{3}}+27 \frac{(x-\tilde{x})^{4}}{35 l^{4}}\right) .
\end{aligned}
$$

Notice that (3) and (4) are the so called Matern $(v=7 / 2$ and $v=9 / 2$ ) formulas respectively. It is worth noting that the family of Matern kernels is a generalization of kernel (2) and it is widely used in multivariate statistical analysis. In this case the absolute exponential kernel is parameterized by an additional parameter $v$. If $v$ goes to infinity then the kernel reduces to Eq. (2), while in the case of $v=1 / 2$ the kernel becomes equivalent to the absolute exponential kernel. Also $\sigma_{f}$ and $l$ are two hyperparameters which can be constrained from the observational data. Since the kernel function plays a role in reconstructing $f(x)$ (in our case comoving distance), we have decided to use the aforementioned kernels in order to test whether the choice of the kernel can affect the amount of the so called $\Lambda \mathrm{CDM}$ cosmokinetic tension.

Here we use the GAPP code [41] in order to reconstruct $f(x)$ and its derivatives. Specifically, $f(x)$ and its derivatives are given by

$$
\begin{aligned}
& f(x) \sim G P(\mu(x), k(x, \tilde{x})) \\
& f^{\prime}(x) \sim G P\left(\mu^{\prime}(x), \frac{\partial^{2} k(x, \tilde{x})}{\partial x \partial \tilde{x}}\right) \\
& f^{\prime \prime}(x) \sim G P\left(\mu^{\prime \prime}(x), \frac{\partial^{4} k(x, \tilde{x})}{\partial^{2} x \partial^{2} \tilde{x}}\right),
\end{aligned}
$$

where $G P$ stands for Gaussian process.

\section{Observational data and method}

The luminosity distance is the ideal tool to investigate the Hubble diagram. Our aim is to extend the Hubble relation to as high redshifts as possible, hence in addition to SNIa, we also consider QSOs and GRBs. In particular, bellow we briefly present the type of standard candles, used in the statistical analysis.

- Supernovae (SNIa): we utilize the "Pantheon" compilation of SNIa data [42]. This sample contains 1048 spectroscopically confirmed SNIa in the redshift range $0.01<z<2.26$.

- Quasars (QSOs): Furthermore, we use the sample of 1598 QSOs as collected by [2,43]. The redshift interval of the current data is $0.04<z<5$. Notice that, in our analysis we use bin-averaged version of QSOs data.

- In addition to the above data, we use a compilation of 162 GRBs [44-46] in the range of $0.03<z<9.3$. Unlike SNIa, QSOs and GRBs are observed up to very high redshifts $(z>3)$ at which the distance modulus is more sensitive to the cosmological parameters [47].

The evolution of the distance modulus is given by $\mu(z)=$ $5 \log D_{L}(z)+25$, hence

$D_{L}(z)=10^{(\mu(z)-25) / 5}$, 
where $D_{L}(z)$ is the luminosity distance from which the normalized comoving distance ${ }^{1}$ is written as

$D(z)=\frac{H_{0}}{c} \frac{D_{L}(z)}{1+z}$.

Notice that $H_{0}$ is the Hubble constant and $c$ is the speed of light.

Based on the above, we compute the normalized comoving distance data points and then we use them in order to reconstruct the form of $D(z)$ as well as its derivatives. As a matter of fact knowing $D(z)$ and its derivatives, it is straightforward to compute the Hubble function $H(z)$ as well as its first and second derivatives, namely

$$
\begin{aligned}
H(z) & =\frac{H_{0}}{D^{\prime}(z)}, \\
H^{\prime}(z) & =-H_{0} \frac{D^{\prime \prime}(z)}{D^{\prime}(z)^{2}}, \\
H^{\prime \prime}(z) & =H_{0}\left[\frac{2 D^{\prime \prime}(z)^{2}}{D^{\prime}(z)^{3}}-\frac{D^{\prime \prime \prime}(z)}{D^{\prime}(z)^{2}}\right] .
\end{aligned}
$$

Moreover using the error propagation we obtain

$$
\begin{aligned}
\delta H(z)= & H_{0} \frac{\delta D^{\prime}(z)}{D^{\prime}(z)^{2}}, \\
\delta H^{\prime}(z)= & H_{0}\left[\frac{\delta D^{\prime \prime}(z)}{D^{\prime}(z)^{2}}-\frac{2 D^{\prime \prime}(z) \delta D^{\prime}(z)}{D^{\prime}(z)^{3}}\right], \\
\delta H^{\prime \prime}(z)= & H_{0}\left[\frac{\delta D^{\prime \prime \prime}(z)}{D^{\prime}(z)^{2}}-\frac{2 D^{\prime \prime \prime}(z) \delta D^{\prime}(z)}{D^{\prime}(z)^{3}}\right. \\
& \left.-\frac{4 D^{\prime \prime}(z) \delta D^{\prime \prime}(z)}{D^{\prime}(z)^{3}}+\frac{6 D^{\prime \prime}(z)^{2} \delta D^{\prime}(z)}{D^{\prime}(z)^{4}}\right] .
\end{aligned}
$$

Notice that in above formula, we use the same $H_{0}$ which has been used to obtain the normalized distance in Eq. (9) and for those quantities with more than one term in uncertainty, we use square root of all terms. For example, for $\delta X=\delta a+\delta b+\delta c+\cdots$, the total uncertainty is $\delta X=\sqrt{(\delta a)^{2}+(\delta b)^{2}+(\delta c)^{2}+\cdots}$.

Following the same notations we compute the deceleration and jerk parameters as well as the corresponding uncertainties. As a function of $D(z)$, these parameters are:

$$
\begin{aligned}
& q(z)=-(1+z) \frac{D^{\prime \prime}(z)}{D^{\prime}(z)}-1 \\
& j(z)=(1+z)^{2}\left[-\frac{D^{\prime \prime \prime}(z)}{D^{\prime}(z)}+3\left(\frac{D^{\prime \prime}(z)}{D^{\prime}(z)}\right)^{2}\right]-2(1+z) \frac{D^{\prime \prime}(z)}{D^{\prime}(z)}+1
\end{aligned}
$$

and as a function of $H(z)$,

$q(z)=(1+z) \frac{H^{\prime}(z)}{H(z)}-1$,

${ }^{1}$ For the rest of the paper $D(x)$ plays the role of $f(x)$.
$j(z)=(1+z)^{2}\left[\frac{H^{\prime \prime}(z)}{H(z)}+\left(\frac{H^{\prime}(z)}{H(z)}\right)^{2}\right]-2(1+z) \frac{H^{\prime}(z)}{H(z)}+1$.

In the case of $\Lambda \mathrm{CDM}$ model, namely $H(z)=H_{0} E(z)=$ $H_{0}\left[\Omega_{m 0}(1+z)^{3}+\Omega_{\Lambda 0}\right]^{1 / 2}$ the cosmokinetic parameters become $q_{\Lambda}(z)=\frac{3}{2} \Omega_{m}(z)-1$ and $j_{\Lambda}(z)=1$, where $\Omega(z)=$ $\Omega_{0}(1+z)^{3} / E(z)^{2}$ and $\Omega_{m 0}+\Omega_{\Lambda 0}=1^{2}$.

Lastly, we remind the reader the basic steps of our method (see Sect. 2). First the normalized distances $D(z)$ data are given as input to the GAPP code [41]. Second we reconstruct the functional form of $D(z)$ and finally we compute the rest of the cosmological quantities. During the process we consider that the aforementioned data-sets can be treated as statistically independent measurements. This assumption is a rather strong statement given that for example the SNIa, QSO and GRB data are sensitive to luminosity distances and there might be spatial overlap between the various probes, hence this could lead to correlations that might affect the statistical analysis. While this is an important point, unfortunately at the moment there is no standard way to account for it given the lack of the full correlation matrix among the different samples. Therefore, following standard lines we have assumed that the different data-sets are uncorrelated. Within this framework, the corresponding parameter space is given by $\left(H_{0}, q_{0}, j_{0}\right)$.

\section{Results and discussion}

In this section, we discuss the main results of our analysis. Specifically, in Table 1 we provide an overall presentation of the cosmographic parameters at the present epoch. In the left panel of Fig. 1 we present the evolution of the reconstructed $D(z)$ and its derivatives when using the Gaussian kernel and SNIa data. As expected $D^{\prime}(z)$ decreases as a function of $z$, hence due to Eq. (10) the Hubble parameter is an increasing function. In the right panel of Fig. 1 we plot the cosmokinetic parameters $H(z) /(1+z), q(z)$ and $j(z)$ as a function of redshift. Moreover in the case of Marten kernels $v=7 / 2$ and $v=9 / 2$ the aforementioned parameters are shown in Fig. 2 , respectively. We observe that the evolution of the kinetic parameters are almost the same with those of Gaussian kernel.

However, when combined SNIa with other probes, such as GRBs, the situation becomes different. Indeed, for SNIa/GRBs we plot in Fig. $3 D(z)$ and its derivatives versus redshift using the Gaussian (left panel) and Matern $v=7 / 2$ (right panel) kernels. For both cases we observe that in the evolution of the corresponding derivatives appears oscillations. It is easy to check that the first derivative of $D(z)$

\footnotetext{
${ }^{2}$ For the $\Lambda$ CDM model we utilize $\Omega_{m 0}=0.3$.
} 

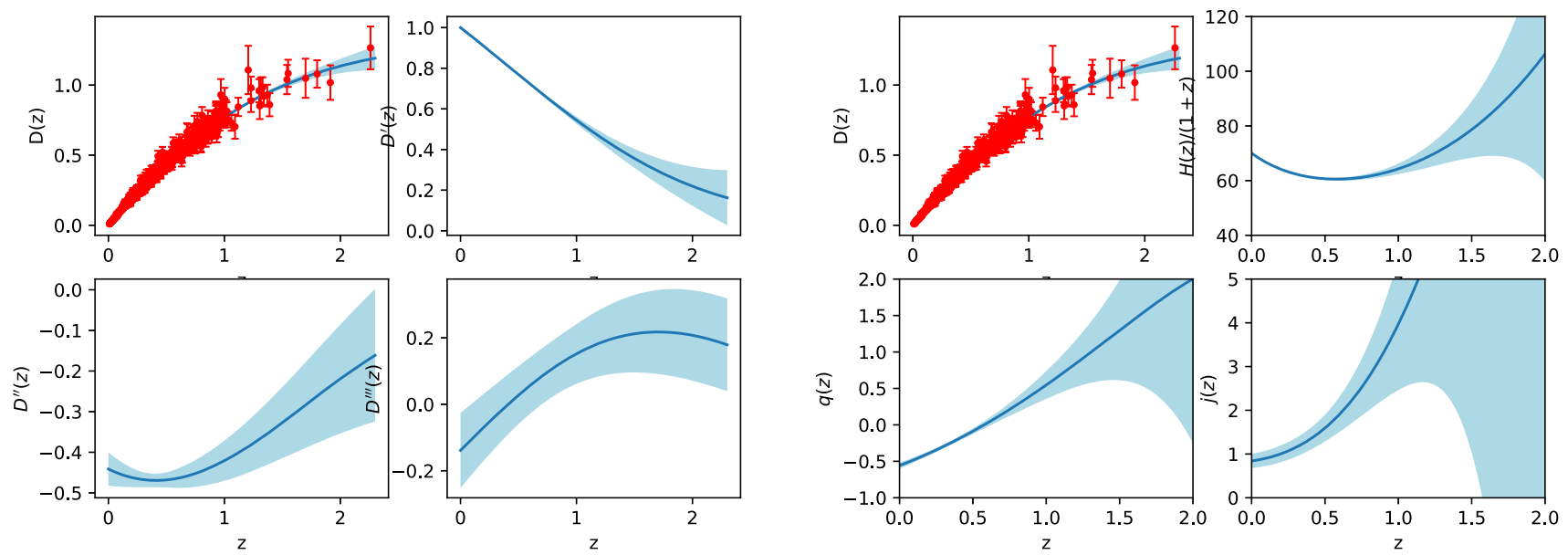

Fig. 1 Left panel: Reconstruction of $D(z)$, data points and its first, second and third derivatives. Right panel: Reconstruction of $D(z)$, the Hubble function, deceleration and jerk parameters as a function of redshift for SNIa data only with the Gaussian kernel
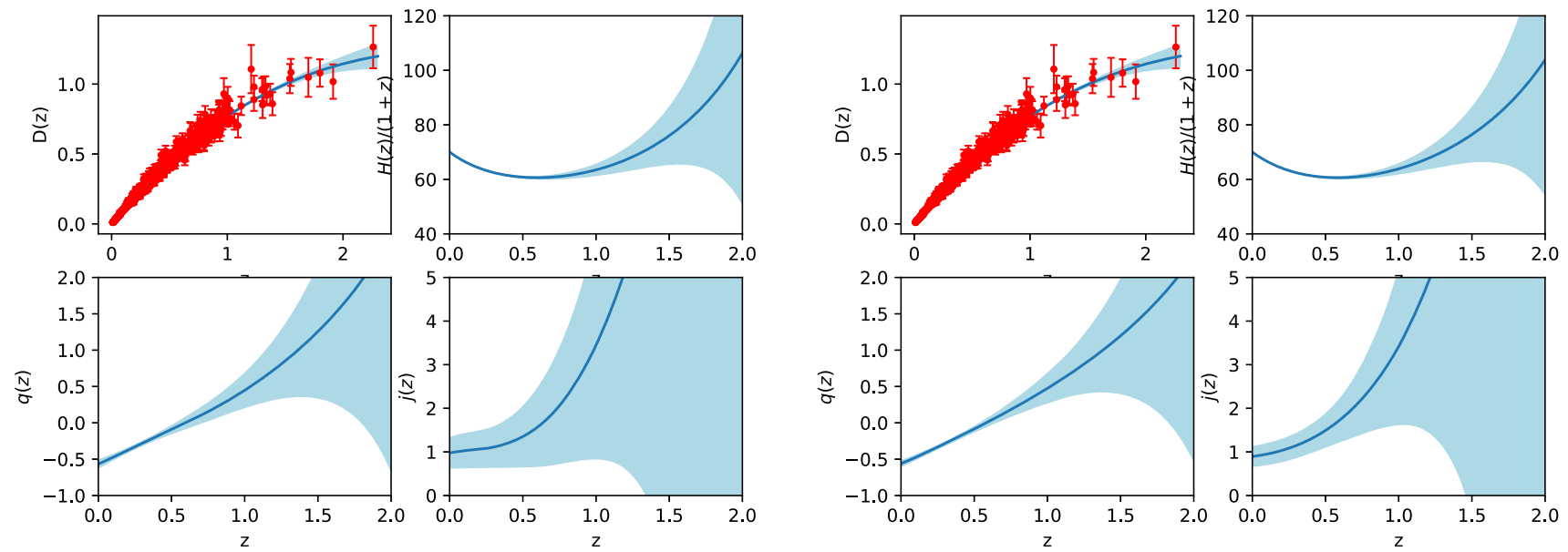

Fig. 2 Left panel: The cosmokinetic parameters as a function of redshift using Matern $(v=7 / 2)$ kernel for SNIa only data. Right panel: The cosmokinetic parameters as a function of redshift using Matern $(v=9 / 2)$ kernel for SN only data
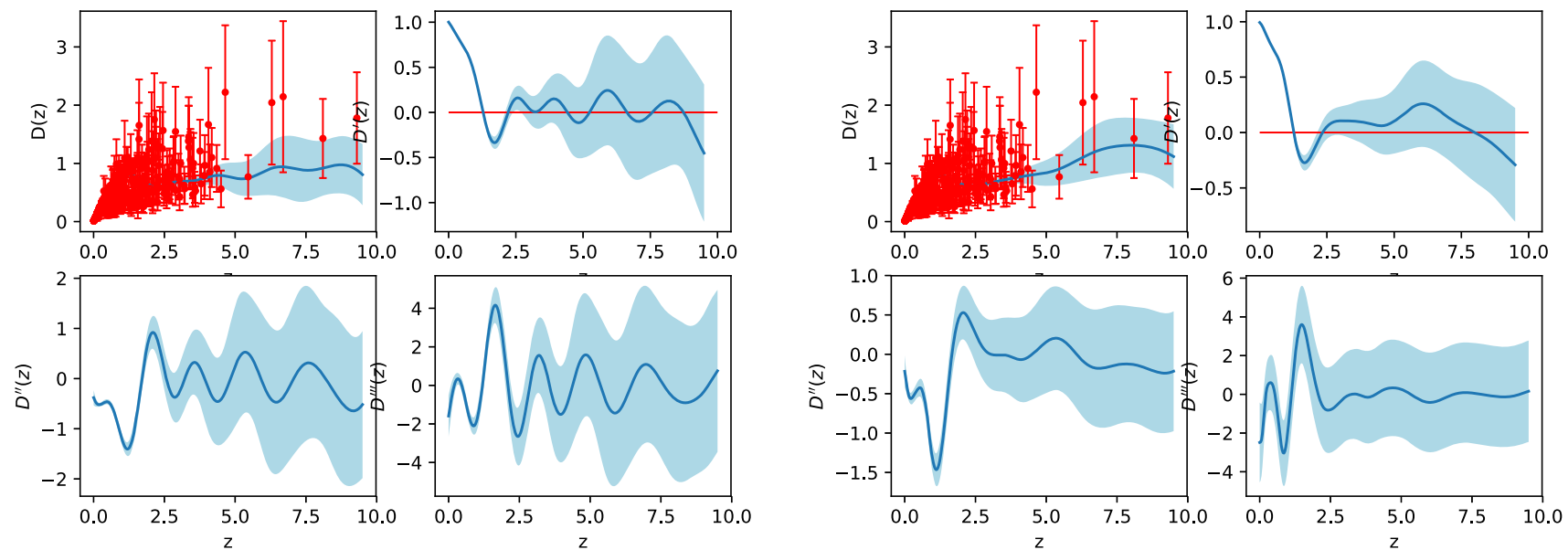

Fig. 3 Left panel: The reconstruction of $D(z)$ and its derivatives as a function of redshift using Gaussian kernel by considering SNIa and GRBs. Right panel: The same plot using Matern $(v=7 / 2)$ kernel and the same data set 

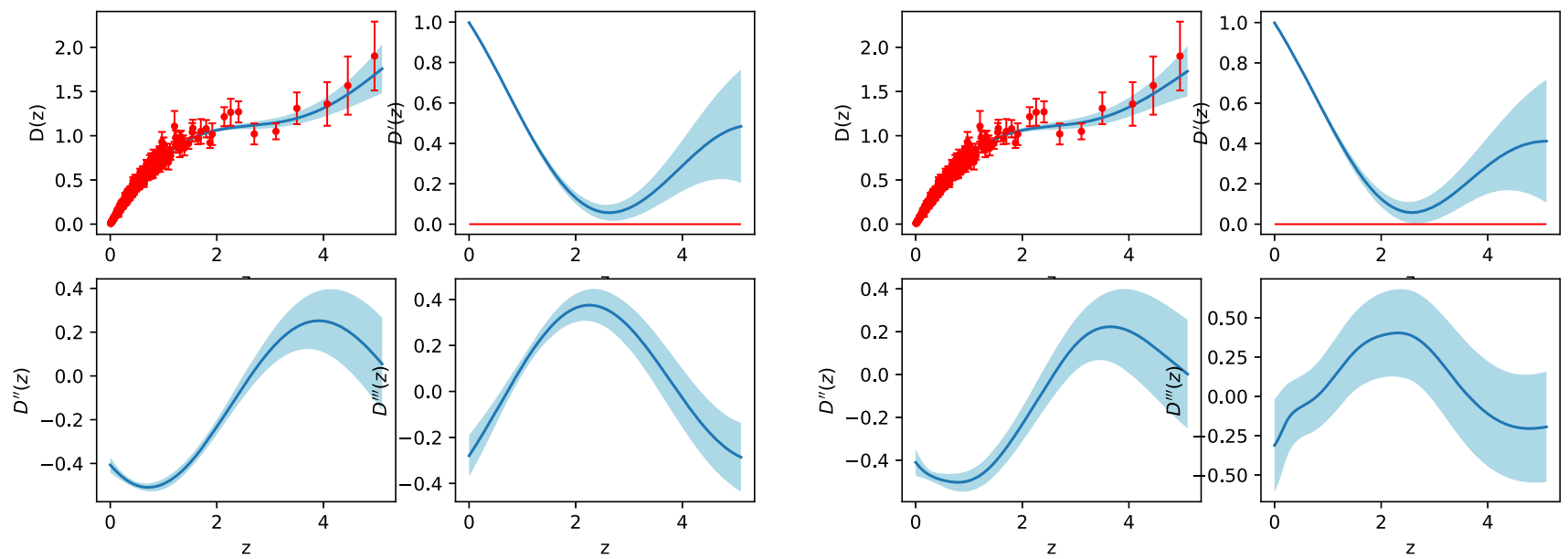

Fig. 4 Left panel: The reconstruction of $D(z)$ and its derivatives as a function of redshift using Gaussian kernel by considering SNIa and QSOs. Right panel: The same plot using Matern $(v=7 / 2)$ kernel and the same data set

Table 1 Cosmokinetic parameters at present time for different data sets and kernels

\begin{tabular}{llll}
\hline Data set Kernel & Gaussian & Matern $v=7 / 2$ & Matern $v=9 / 2$ \\
\hline SNIa & $H_{0}=70.00 \pm 0.38$ & $H_{0}=69.98 \pm 0.46$ & $H_{0}=69.98 \pm 0.42$ \\
& $q_{0}=-0.558 \pm 0.04$ & $q_{0}=-0.567 \pm 0.06$ & $q_{0}=-0.561 \pm 0.05$ \\
& $j_{0}=0.84 \pm 0.16$ & $j_{0}=0.98 \pm 0.36$ & $j_{0}=0.89 \pm 0.23$ \\
SNIa+GRBs & $H_{0}=69.92 \pm 0.72$ & $H_{0}=70.49 \pm 0.85$ & $H_{0}=70.28 \pm 0.77$ \\
& $q_{0}=-0.62 \pm 0.15$ & $q_{0}=-0.79 \pm 0.20$ & $q_{0}=-0.73 \pm 0.17$ \\
& $j_{0}=2.26 \pm 1.1$ & $j_{0}=3.21 \pm 2.1$ & $j_{0}=2.80 \pm 1.4$ \\
SNIa+QSOs & $H_{0}=70.17 \pm 0.35$ & $H_{0}=70.13 \pm 0.45$ & $H_{0}=70.15 \pm 0.40$ \\
& $q_{0}=-0.59 \pm 0.03$ & $q_{0}=-0.58 \pm 0.06$ & $q_{0}=-0.59 \pm 0.05$ \\
SNIa+GRBs+QSOs & $j_{0}=0.96 \pm 0.13$ & $j_{0}=0.99 \pm 0.33$ & $j_{0}=0.95 \pm 0.21$ \\
& $H_{0}=70.86 \pm 0.42$ & $H_{0}=70.22 \pm 0.69$ & $H_{0}=70.12 \pm 0.63$ \\
& $q_{0}=-0.72 \pm 0.05$ & $q_{0}=-0.66 \pm 0.14$ & $q_{0}=-0.62 \pm 0.11$ \\
& $j_{0}=1.62 \pm 0.2$ & $j_{0}=1.98 \pm 1.2$ & $j_{0}=1.55 \pm 0.81$ \\
\hline
\end{tabular}

crosses the zero line several times, hence the cosmokinetic parameters diverge at these points. Notice that utilizing the Matern $v=9 / 2$ kernel the results remain unaltered. We argue that although GRBs may help to reconstruct the cosmic expansion up to $z \sim 10$, however there are practical difficulties in achieving this goal in the case of Gaussian process.

Moreover, the results of SNIa/QSO combination are presented in Fig. 4 and Table 1. In this case, we observe that $D(z)$ slowly decreases prior to $z \sim 3$, hence a small oscillation appears at that redshift. Furthermore, we find that both Gaussian and Matern kernels provide similar results and in contrast to SNIa/GRB case, here the first derivative of the $D(z)$ does not cross the zero line at $1 \sigma$ level.

Lastly, we combine SNIa, GRBs and QSOs in order to compute the reconstructed comoving distance for all kernels. As an example in Fig. 5 we plot the evolution of $D(z)$ and the corresponding derivatives in the case of Matern $v=7 / 2$ kernel. Again we verify that there are epochs which are located at large redshifts and for which $D^{\prime}(z)$ crosses the zero line (similar behavior is found for the other kernels).

Now we focus on Table 1 which shows the cosmokinetic parameters at the present time for various data and kernels explored in this study. Considering only the traditional standard candles (SNIa), we find that the Hubble constant is close to $70 \mathrm{Km} / \mathrm{s} / \mathrm{Mpc}$ regardless the form of kernel, while $q_{0}$ and $j_{0}$ are consistent (within $1 \sigma$ ) with those of $\Lambda \mathrm{CDM}$. Combining SNIa and GRB data, we find that the value of $H_{0}$ does not change significantly and it remains close to $70 \mathrm{Km} / \mathrm{s} / \mathrm{Mpc}$. In the case of Gaussian kernel, the current value of the deceleration parameter is in agreement with that of $\Lambda \mathrm{CDM}$ at 1 $\sigma$ level. For the Matern's kernels the extracted value of $q_{0}$ is marginally consistent with $\Lambda \mathrm{CDM}$ with $q_{0}<q_{\Lambda, 0}$. Concerning $j_{0}$, our results are similar to those of [1], however the corresponding uncertainties are larger (by a factor of 2.5-4) than those of [1], implying that the extracted jerk parameters are consistent with the the predictions of $\Lambda \mathrm{CDM}$ at $2 \sigma$ level. Combining SNIa and QSO datasets, we find that for all 

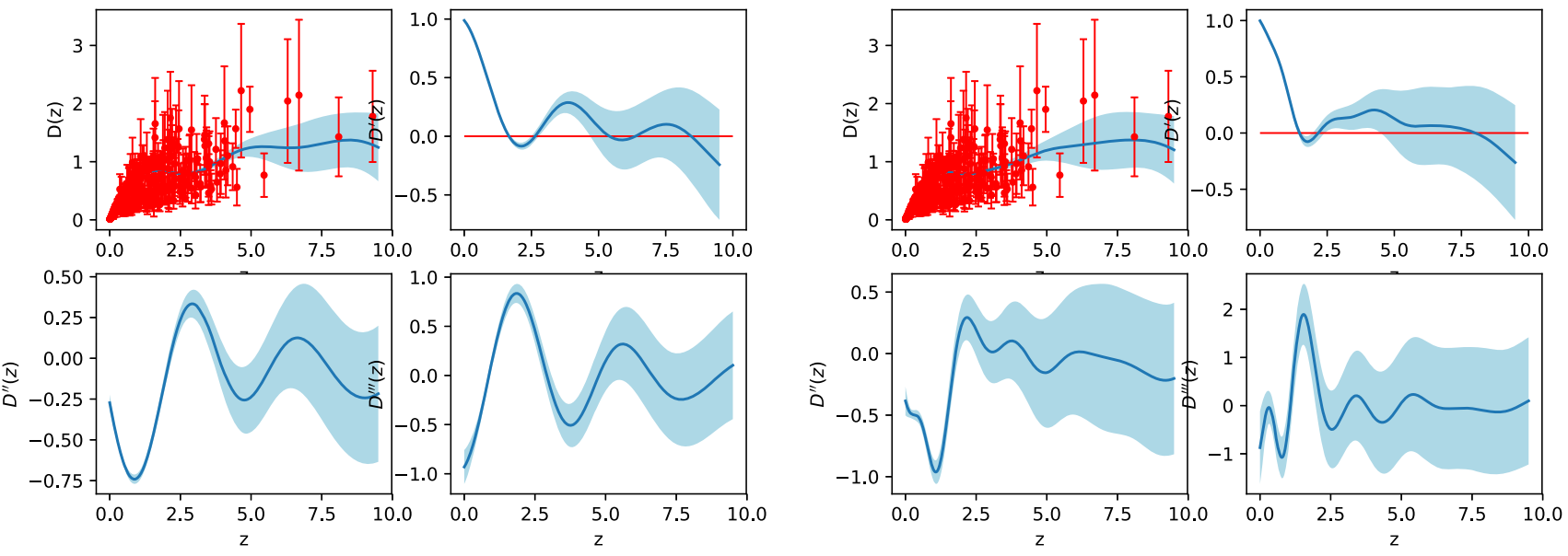

Fig. 5 Left panel: The reconstruction of $D(z)$ and its derivatives as a function of redshift using Gaussian kernel for all data set. Right panel: The same plot using Matern $(v=7 / 2)$ kernel and the same data set
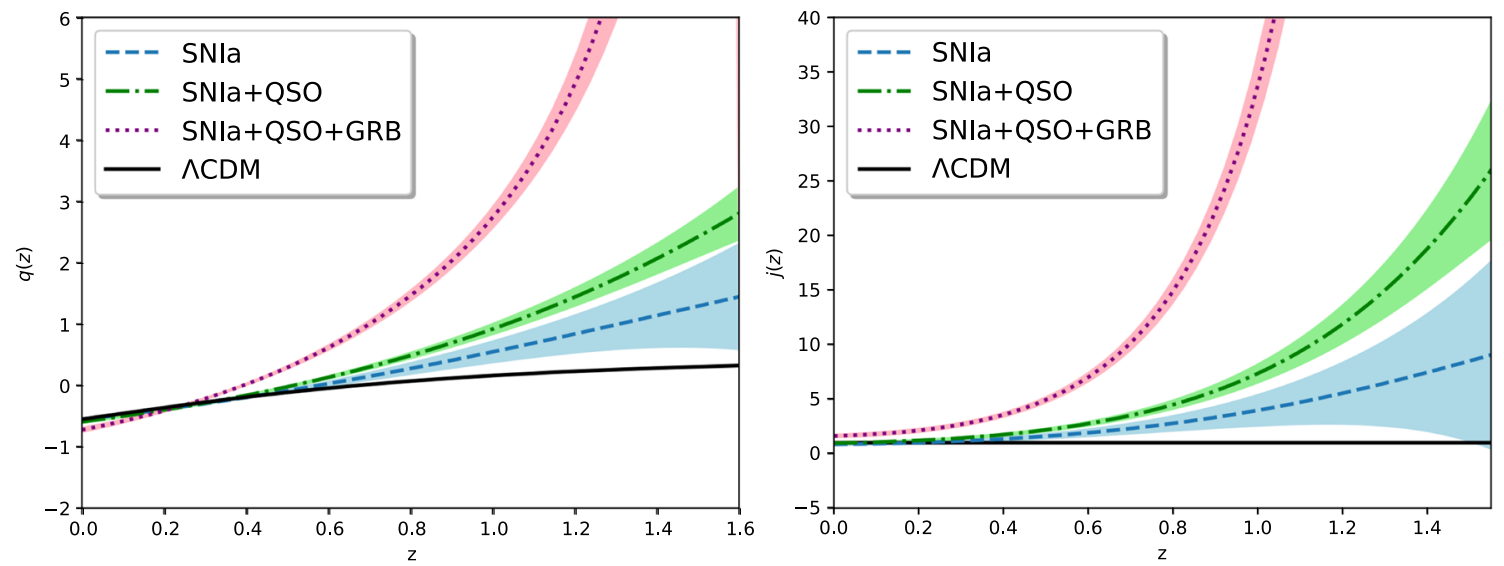

Fig. 6 Left panel: Reconstruction of $q(z)$ using different data sets and considering the Gaussian kernel. Right panel: Reconstruction of $j(z)$. In the case of $\Lambda \mathrm{CDM}$ model we use $\Omega_{m 0}=0.3$ (see solid black lines)

kernels the cosmokinetic parameters $\left(q_{0}, j_{0}\right)$ are in a good agreement (with $1 \sigma$ ) with those of $\Lambda \mathrm{CDM}$ model.

Finally, in the case of the Gaussian kernel the combination SNIa/QSOs/GRBs indicates that the extracted values of $q_{0}$ and $j_{0}$ are $\sim 3 \sigma$ away from those of $\Lambda \mathrm{CDM}$. However, the opposite situation holds in the case of Matern's kernels, namely both $q_{0}$ and $j_{0}$ are consistent (due to large uncertainties) with the predictions of $\Lambda \mathrm{CDM}$. In a nutshell, for the usual standard candles (SNIa data) and for the combination SNIa/QSOs we find that the cosmokinetic parameters $\left(q_{0}, j_{0}\right)$ extracted from the Gaussian process are consistent with $\Lambda \mathrm{CDM}$. However, including GRBs in the analysis we find a tension of the $\Lambda \mathrm{CDM}$ model which lies between 2 and $3 \sigma$ levels respectively. Moreover, the combined SNIa/QSO/GRB analysis shows that the choice of the kernel function might affect the amount of tension. Indeed in the case of Matern's kernels we produce cosmokinetic parameters which are consistent with those of $\Lambda \mathrm{CDM}$, while using the Gaussian kernel it seems that the $\Lambda \mathrm{CDM}$ model is in tension with the measurements $\left(q_{0}, j_{0}\right)$.

\subsection{Cosmokinetic parameters at high redshits}

Apart from $\left(q_{0}, j_{0}\right)$ it is useful to study the cosmokinetics parameters at high redshifts. For the Gaussian kernel we plot in Fig. 6 the evolution of $q(z)$ and $j(z)$ in the case of SNIa (blue dashed line), SNIa/QSO (green dot-dashed) and SNIa/QSO/GRBs (magenta dotted curve). For comparison we also plot $q_{\Lambda}(z)$ and $j_{\Lambda}(z)$ (see solid lines). Since $D^{\prime}(z)$ may cross the zero line prior to $z \sim 2$ we prefer to focus on $1<z<2$. Obviously, a strong deviation from the $\Lambda \mathrm{CDM}$ predictions is observed in the case of $\mathrm{SNIa} / \mathrm{QSO}$ and SNIa/QSO/GRBs. We also checked that this result persists regardless the form of the kernel. Although the situation regarding the cosmokinetic tension is not so clear in the present epoch, at high redshifts there is a clear indica- 
tion that such a tension really exists. Especially, the jerk parameter clearly points to this direction, hence the possibility of having new Physics is not excluded by the present analysis. Notice that, our results are in agreement with those of $[1,2]$ who found that the deviation from the flat $\Lambda \mathrm{CDM}$ becomes strong at high redshifts $(z>1)$. Combining our model-independent parametrization of the Hubble Diagram with those of [1,2] we conclude that the deviation from the concordance $\Lambda \mathrm{CDM}$ model is due to new Physics.

\section{Conclusion}

It is well known that the concordance $\Lambda \mathrm{CDM}$ model fits accurately the current cosmological data [7], nonetheless it has been proposed that the model is not without its problems. Indeed there are indications that the $\Lambda \mathrm{CDM}$ model is in tension with some important measurements $[32,33]$, namely the Hubble constant $H_{0}$ and the present value of the mass variance at $8 h^{-1} \mathrm{Mpc}$, namely $\sigma_{8}$. In this context, Lusso et al. [1] using a combined Hubble diagram of SNIa, Quasars, and Gamma-Ray Bursts (GRBs) found a $\sim 4 \sigma$ tension between the best fit cosmokinetic parameters with respect to those of $\Lambda \mathrm{CDM}$ (see also [2]). Whether the above tensions are the result of yet unknown systematic errors or indicate some underlying new Physics is still an open issue. Therefore, on this subject an intense debate is taking place in the literature and the aim of the present work is to contribute to this debate.

In particular, we combined the traditional standard candles (SNIa data) with other extragalactic sources (Quasars and GRBs) to reconstruct, in a model independent way, the Hubble diagram to as high redshifts as possible and to compute the corresponding cosmokinetic parameters at the present epoch, namely deceleration $q_{0}$ and jerk $j_{0}$ parameters. Using only the SNIa data we found that the cosmokinetic parameters $\left(q_{0}, j_{0}\right)$ extracted from the Gaussian process are consistent with those of $\Lambda \mathrm{CDM}$. Also in the case of SNIa/QSO combination, we found that for all kernels the cosmokinetic parameters are in a very good agreement (with $1 \sigma$ ) with those of $\Lambda \mathrm{CDM}$ model.

On the other hand combining SNIa with Quasars and GRBs we revealed some tension, which lies between $2 \sigma$ and $3 \sigma$ levels, depending on the kernel choice. Finally, focusing our analysis on high redshifts $(z>1)$ we found that the corresponding cosmokinetic parameters significantly deviate from those of $\Lambda \mathrm{CDM}$. Overall the combination of the present work with those of $[1,2]$ provide a complete investigation of the so called $\Lambda \mathrm{CDM}$ "tension". The three works, which are model independent, clearly suggest that the discrepancy between the Hubble diagram data (especially for $z>1$ ) and the pre- dictions of the concordance $\Lambda \mathrm{CDM}$ model is the result of some underlying new Physics.

Data Availability Statement This manuscript has no associated data or the data will not be deposited. [Authors' comment: All data used in this work are publicly available. See given references All tables and figures are included.]

Open Access This article is licensed under a Creative Commons Attribution 4.0 International License, which permits use, sharing, adaptation, distribution and reproduction in any medium or format, as long as you give appropriate credit to the original author(s) and the source, provide a link to the Creative Commons licence, and indicate if changes were made. The images or other third party material in this article are included in the article's Creative Commons licence, unless indicated otherwise in a credit line to the material. If material is not included in the article's Creative Commons licence and your intended use is not permitted by statutory regulation or exceeds the permitted use, you will need to obtain permission directly from the copyright holder. To view a copy of this licence, visit http://creativecomm ons.org/licenses/by/4.0/.

Funded by SCOAP ${ }^{3}$.

\section{References}

1. E. Lusso, E. Piedipalumbo, G. Risaliti, M. Paolillo, S. Bisogni, E. Nardini, L. Amati, Astron. Astrophys. 628, L4 (2019). https://doi. org/10.1051/0004-6361/201936223

2. G. Risaliti, E. Lusso, Nat. Astron. 3(3), 272 (2019). https://doi.org/ 10.1038/s41550-018-0657-z

3. A.G. Riess, A.V. Filippenko, P. Challis et al., AJ 116, 1009 (1998)

4. S. Perlmutter, G. Aldering, G. Goldhaber et al., ApJ 517, 565 (1999)

5. E. Komatsu, K.M. Smith, J. Dunkley et al., ApJS 192, 18 (2011)

6. X.I.V. Planck Collaboration, Astron. Astrophys. 594, A14 (2016)

7. N. Aghanim, et al., (2018)

8. D.J. Eisenstein et al., ApJ 633, 560 (2005). https://doi.org/10.1086/ 466512

9. W.J. Percival, B.A. Reid, D.J. Eisenstein et al., MNRAS 401, 2148 (2010)

10. C. Blake et al., Mon. Not. Roy. Astron. Soc. 415, 2876 (2011). https://doi.org/10.1111/j.1365-2966.2011.18903.x

11. B.A. Reid, L. Samushia, M. White, W.J. Percival, M. Manera et al., MNRAS 426, 2719 (2012). https://doi.org/10.1111/j.1365-2966. 2012.21779.x

12. T.M.C. Abbott et al., Mon. Not. Roy. Astron. Soc. 483(4), 4866 (2019). https://doi.org/10.1093/mnras/sty3351

13. S. Alam et al., Mon. Not. Roy. Astron. Soc. 470(3), 2617 (2017). https://doi.org/10.1093/mnras/stx721

14. H. Gil-Marín et al., Mon. Not. Roy. Astron. Soc. 477(2), 1604 (2018). https://doi.org/10.1093/mnras/sty453

15. O. Farooq, F.R. Madiyar, S. Crandall, B. Ratra, Astrophys. J. 835(1), 26 (2017). https://doi.org/10.3847/1538-4357/835/1/26

16. S. Weinberg, Rev. Modern Phys. 61, 1 (1989). https://doi.org/10. 1103/RevModPhys.61.1

17. P. Peebles, B. Ratra, Rev. Mod. Phys. 75, 559 (2003). https://doi. org/10.1103/RevModPhys.75.559

18. E.J. Copeland, M. Sami, S. Tsujikawa, IJMP D15, 1753 (2006). https://doi.org/10.1142/S021827180600942X

19. T. Chiba, S. Dutta, R.J. Scherrer, Phys. Rev. D 80, 043517 (2009). https://doi.org/10.1103/PhysRevD.80.043517

20. L. Amendola, S. Tsujikawa, Dark Energy: Theory and Observations (Cambridge University Press, Cambridge, 2010) 
21. A. Mehrabi, Phys. Rev. D 97(8), 083522 (2018). https://doi.org/ 10.1103/PhysRevD.97.083522

22. A. Mehrabi, S. Basilakos, Eur. Phys. J. C 78(11), 889 (2018). https://doi.org/10.1140/epjc/s10052-018-6368-x

23. H.J. Schmidt, Astron. Nachr. 311, 165 (1990)

24. G. Magnano, L.M. Sokolowski, Phys. Rev. D 50, 5039 (1994). https://doi.org/10.1103/PhysRevD.50.5039

25. A. Dobado, A.L. Maroto, Phys. Rev. D 52, 1895 (1995). https:// doi.org/10.1103/PhysRevD.52.1895

26. S. Capozziello, S. Carloni, A. Troisi, Recent Res. Dev. Astron. Astrophys. 1, 625 (2003)

27. S.M. Carroll, V. Duvvuri, M. Trodden, M.S. Turner, Phys. Rev. D 70, 043528 (2004). https://doi.org/10.1103/PhysRevD.70.043528

28. S. Weinberg, Rev. Modern Phys. 61(1), 1 (1989). https://doi.org/ 10.1103/RevModPhys.61.1

29. T. Padmanabhan, Phys. Rep. 380(5-6), 235 (2003). https://doi.org/ 10.1016/S0370-1573(03)00120-0

30. L. Perivolaropoulos. Six puzzles for lcdm cosmology (2008)

31. A. Padilla. Lectures on the cosmological constant problem (2015)

32. L. Verde, T. Treu, A.G. Riess, Nat. Astron. 3(10), 891-895 (2019). https://doi.org/10.1038/s41550-019-0902-0

33. J. Solà, A. Gómez-Valent, J. de Cruz Pérez, Physics Letters B 774, 317 (2017). https://doi.org/10.1016/j.physletb.2017.09.073

34. M. Rezaei, M. Malekjani, J. Solà Peracaula, Phys. Rev. D 100(2), 023539 (2019). https://doi.org/10.1103/PhysRevD.100.023539

35. J. Solà Peracaula, A. Gómez-Valent, J. de Cruz Pérez, C. Moreno-Pulido, ApJ 886(1), L6 (2019). https://doi.org/10.3847/ 2041-8213/ab53e9

36. H. Velten, S. Gomes, Phys. Rev. D 101, 043502 (2020). https://doi. org/10.1103/PhysRevD.101.043502
37. K. Liao, A. Shafieloo, R.E. Keeley, E.V. Linder, (2019)

38. M.J. Zhang, H. Li, Eur. Phys. J. C 78(6), 460 (2018). https://doi. org/10.1140/epjc/s10052-018-5953-3

39. A. Gómez-Valent, L. Amendola, J. Cosmol. Astropart. Phys. 2018(4), 051 (2018). https://doi.org/10.1088/1475-7516/2018/04/ 051

40. F. Melia, M.K. Yennapureddy, JCAP 1802(02), 034 (2018). https:// doi.org/10.1088/1475-7516/2018/02/034

41. M. Seikel, C. Clarkson, M. Smith, JCAP 1206, 036 (2012). https:// doi.org/10.1088/1475-7516/2012/06/036

42. D.M. Scolnic et al., Astrophys. J. 859(2), 101 (2018). https://doi. org/10.3847/1538-4357/aab9bb

43. G. Risaliti, E. Lusso, Astrophys. J. 815, 33 (2015). https://doi.org/ 10.1088/0004-637X/815/1/33

44. M. Demianski, E. Piedipalumbo, D. Sawant, L. Amati, Astron. Astrophys. 598, A113 (2017). https://doi.org/10.1051/0004-6361/ 201628911

45. M. Demianski, E. Piedipalumbo, D. Sawant, L. Amati, Astron. Astrophys. 598, A112 (2017). https://doi.org/10.1051/0004-6361/ 201628909

46. L. Amati, M. Della Valle, Int. J. Mod. Phys. D22(14), 1330028 (2013). https://doi.org/10.1142/S0218271813300280

47. M. Plionis, R. Terlevich, S. Basilakos, F. Bresolin, E. Terlevich, J. Melnick, R. Chavez, Mon. Not. Roy. Astron. Soc. 416, 2981 (2011). https://doi.org/10.1111/j.1365-2966.2011.19247.x 